\title{
Brownian Tomography of Biomolecules and Soft Polymer Assemblies
}

Cesare De Pace $^{1}$, Gabriele MArchello ${ }^{1}$, Lorena Ruiz Perez ${ }^{1}$ and Giuseppe Battaglia ${ }^{2}$

${ }^{1}$ University College London (UCL), London, England, United Kingdom, ${ }^{2}$ University College of London (UCL); Institute for Bioengineering of Catalonia (IBEC); The Barcelona Institute for Science and Technology (BIST); Institució Catalana de Recerca i Estudis Avançats (ICREA), London, England, United Kingdom

One of the most powerful techniques for structural determination is electron microscopy (EM), which is able to achieve imaging at atomic resolution. However, EM requires keeping the beam under high vacuum to avoid undesired scattering in the electron path. This drawback leads to solidification techniques (such as controlled drying cryogenic techniques), which may alter the microstructure and chemical nature of the sample. The mesoscopic structure of biological and soft specimens is controlled by the presence of their media. Fast vitrification overcame these drawbacks; yet, cryogenic TEM works on static snapshots and does not allow the observation of dynamic events.

In our approach, we use liquid-phase EM (LPEM) to investigate structures in their native liquid state and monitor dynamic processes. An efficient approach for EM imaging of materials in liquid form is achieved by enclosing the liquid-state sample within electron-transparent membranes which shield the liquid medium and the high vacuum of the microscope column [1]. LPEM offers tremendous potential in many different fields ranging from soft matter, nanomaterials, polymer assemblies, biomaterials, synthetic biology, design and catalysis to name a few. There are two important limitations arising from the liquid nature of the sample: (i) similar to cryogenic TEM, the imaging contrast can be considerably hindered by the liquid thickness; (ii) the electron beam may affect the material under study. Thus, the need for lowdose imaging conditions becomes imperative.

The liquid nature of the specimen, however, bestows exciting new opportunities. Particles dispersed in a liquid undergo continuous translational displacement and rotation, called Brownian motion. Such motion leads each particle dispersed in the liquid to display several profiles under the TEM, potentially, disclosing all of its surfaces. We have exploited this feature for investigating different soft organic systems in liquid: (i) a polymer assembly with a localised asymmetry, and (ii) several biomolecules (e.g. ferritin). Both systems were imaged over time with a high frame-rate-acquisition direct detection in-situ camera from Gatan, the K2-IS. This component becomes pivotal to enable high contrast and resolution (spatial and temporal). Thus, a high variety of particle profiles were produced over time; hence, making time a fundamental element of the reconstruction process. Our method set the basis for Brownian tomography, $3 \mathrm{D}$ reconstructions taking advantage of the Brownian motion. This technique promises great potential for time-resolved 3D structure reconstruction of fully hydrated soft specimens in their native environment. 

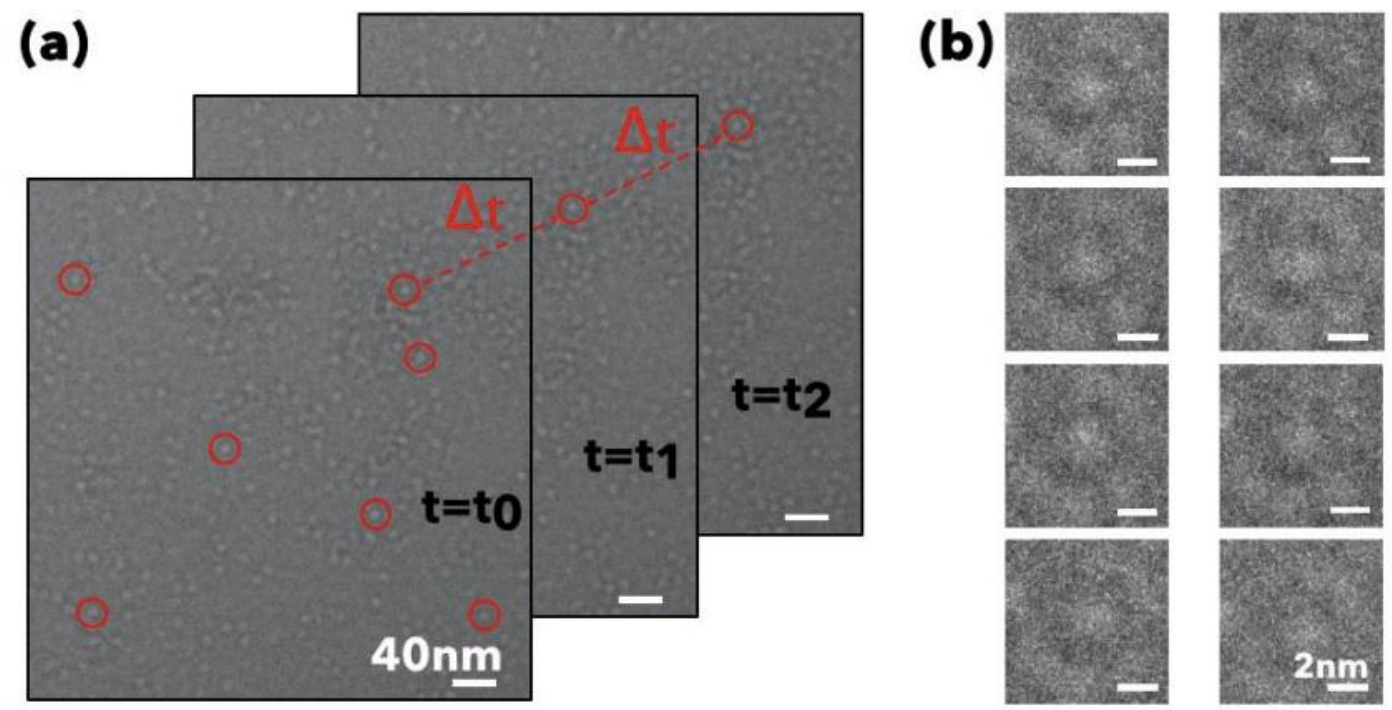

Figure 1. Schematic diagram showing the principle of Brownian tomography of ferritin. a) a single particle of ferritin being tracked across three consecutive frames and b) various profiles of the same ferritin particles with their associated angular orientations obtained from eight consecutive frames

(a)

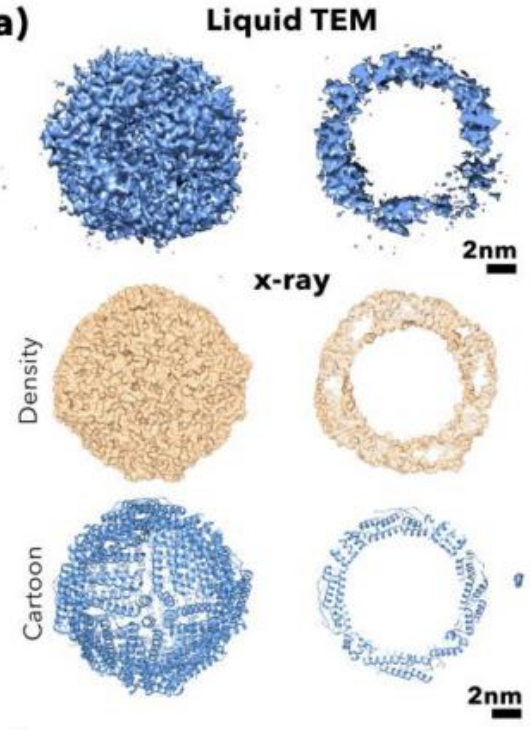

(b) Liquid TEM x-ray

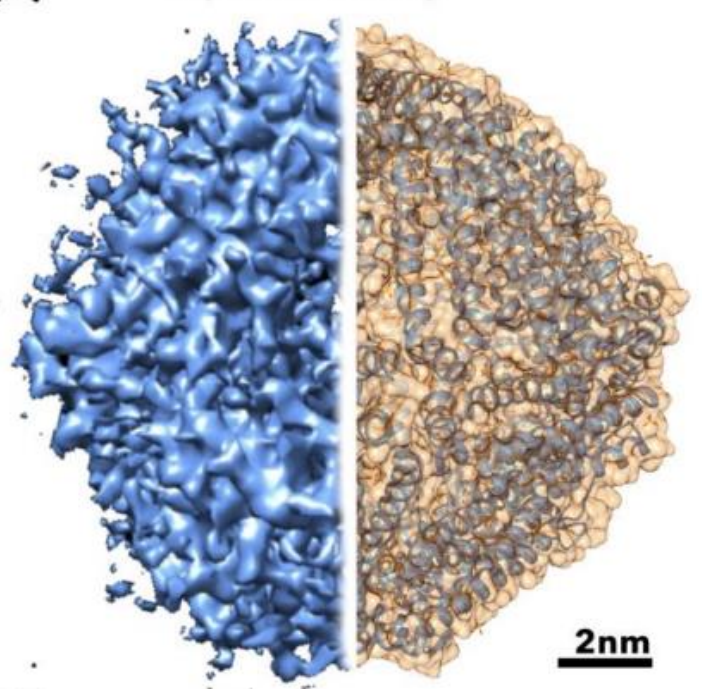

Figure 2. Comparison between liquid TEM and $\mathrm{x}$-ray reconstructed $3 \mathrm{D}$ density map (in blue) of ferritin and the ground truth (in orange). The density map of ferritin was while the 3D model was (a) 3D density map generated by Brownian tomography and the model downloaded from the protein database (PDB) file:3F32 and reconstructed from X-ray diffraction. The models are displayed both as top view and the equatorial section with the $\mathrm{x}$-ray reconstructed structure shown as density map and ribbon cartoon to highlight the protein folding. (b) Halves of the reconstructed 3D map and model of ferritin displayed together highlighting the similarities between both structures. The size is comparable, and the pattern of the helixes in the ground truth continues in the noisy pattern of the reconstructed density map.

\section{References}

Marchello, G.*, De Pace, C.*, Wilkinson, N., Ruiz-Perez, L. \& Battaglia, G. 4D Liquid-phase Electron Microscopy of Ferritin by Brownian Single Particle Analysis. (2019).arXiv: 1907.03348 